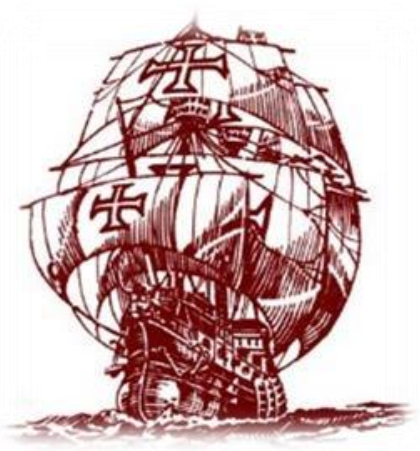
Nau Literária: crítica e teoria de literaturas
www.seer.ufrgs.br/nauliteraria
ISSN 1981-4526 - PPG-LET-UFRGS - Porto Alegre
Vol. 11 N. $01-2015$
100 anos da geração Orpheu

\title{
Expediente do número
}

\section{Editores}

Márcia Ivana de Lima e Silva, UFRGS

Milton Colonetti, PUCRS

Guilherme Valls Darisbo, UFOP

\section{Comissão editorial executiva}

Maurício dos Santos Gomes, UFRGS

\section{Editoração Digital}

Daniel Menezes Luz, UFRGS

Milton Colonetti, PUCRS

\section{Revisão geral}

Guilherme Valls Darisbo, UFOP

Daniel Menezes Luz, UFRGS

\section{Revisores desta edição}

Bruno Pommer, UFRGS

Daniel Menezes Luz, UFRGS

Gisélle Razera, UFRGS

Guilherme Valls Darisbo, UFOP

Luciana Gouvêa da Cunha, UFOP

Sandra Beatriz Salenave de Brito, UFRGS / IFSul

\section{Conselho editorial}

Ana Lúcia Tettamanzy, UFRGS

Ana Maria Lisboa de Mello, PUCRS

Andrea Perrot, UFPEL

Anselmo Peres Alós, UFSM

Atílio Bergamini Junior, UFRGS 
Cláudia Mentz Martins, FURG

Denise Dumith, FURG

Eliana Pritsch, FAPA, UNISINOS

Gínia Maria Gomes, UFRGS

Gustavo Rückert, UNIPAMPA

Inara de Oliveira Rodrigues, UESC

Jaime Ginzburg, USP

Juracy Saraiva, FEEVALE

Lilian C. de Campos Martinez, UNAM (México)

Lucia Regina Lucas Rosa, UNILASALLE

Luiza Vilma Pires Vale, FAPA

Marcia Barbosa, UPF

Márcia Ivana de Lima e Silva, UFRGS

Marcia Lopes Duarte, UNISINOS

Marcus Rogerio Salgado, UFRJ

Maria da Glória Bordini, UFRGS

Maurício dos Santos Gomes, UFRGS

Mauro Nicola Póvoas, FURG

Michel Peterson, UdeM

Milton Colonetti, PUCRS

Naduska Palmeira, IST-STP / UNIABEU

Paulo Roberto Alves, UFSM

Pauly Ellen Bothe, UNAM (México)

Pedro Mandagará, UERR

Priscilla de Oliveira Ferreira, UFRGS

Regina da Costa, UNIRITTER

Regina Zilberman, UFRGS

Rita de Cássia Cavalcante, UFRGS

Rita Lenira Bittencourt, UFRGS

Ruben Daniel Castiglioni, UFRGS

Sandra Maggio, UFRGS

Seleste Michels Rosa, UFRGS

Sérgio Wladimir Cazé dos Santos, UFES

Silvia Niederauer, UNIFRA 
Silvia Paraense, UFSM

Terezinha Marlene Lopes Teixeira, UNISINOS

Vera Haas, IFSul

Vera Medeiros, UNIPAMPA 